ISSN 1991- 8690

website : http:// jsci.utq.edu.iq

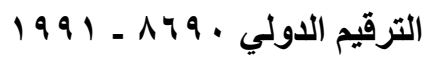

Email: utjsci@utq.edu.iq

\title{
Studying Effect of Solar Concentrator Type on Small Signal Gain for Pumping Nd: YAG Crystal
}

\author{
Mohanad M. Azzawi \\ Hisham A. Maliek* \\ Bushra R. Mahdi \\ Laser and Optoelectronics Center- Ministry of science \& Technology \\ *hushamabid@yahoo.com
}

\begin{abstract}
$\underline{\text { Abstract }}$
In this research, solar flux is applied as a source for pumping Nd:YAG crystal. Lasing action could be gained when there is enough pumped solar flux expressed in term of small signal gain, SSG. Concentration ratio is the term of determining how the solar flux is close to produce SSG for lasing action. Concentration is achieved by applying a concentrator. Two types of concentrators are applied for comparison: Frensel lens and parabolic reflecting dish. It is found that, a solar intensity of $800 \mathrm{~W} / \mathrm{m}^{2}$ incident on Frensel lens of aperture area (729) $\mathrm{Cm}^{2}$ has a concentration ratio of (18000) compared with a parabolic reflecting dish of aperture area (7065) $\mathrm{Cm}^{2}$ has a concentration ratio of (1000). It is clear that Frensel lens is preferred over parabolic reflecting dish because it meets the required value of SSG for lasing Nd:YAG crystal.
\end{abstract}

Keywords: concentration ratio, dish, Fresnel lens, small signal gain.

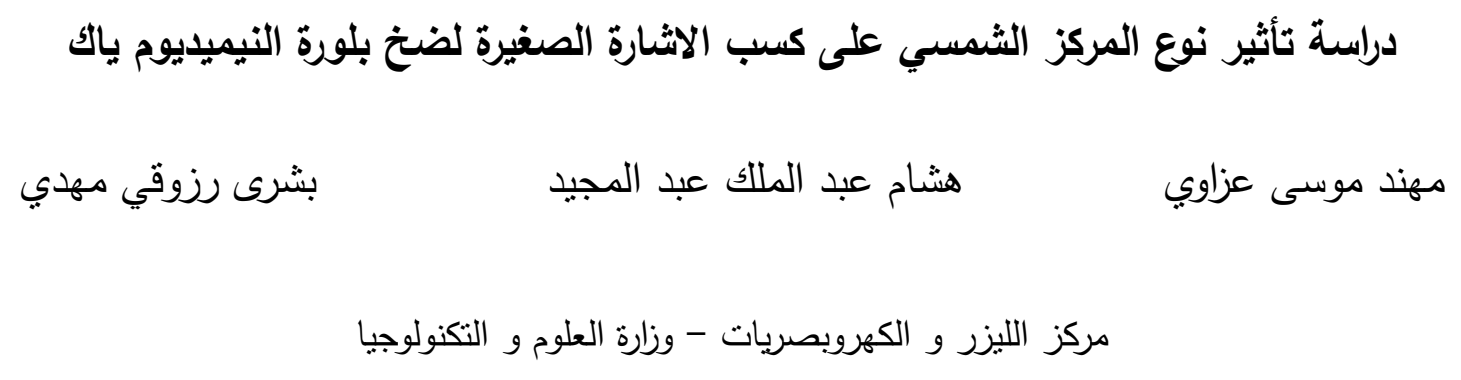

$\underline{\text { الخلاصة }}$

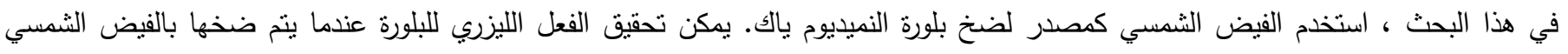

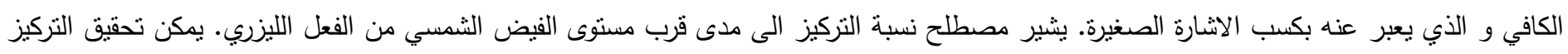

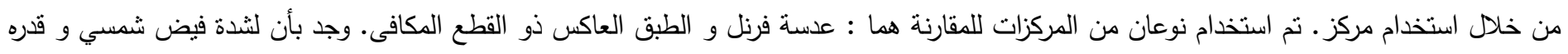

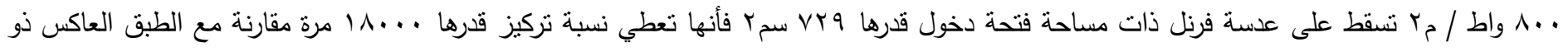

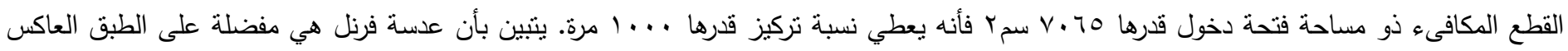

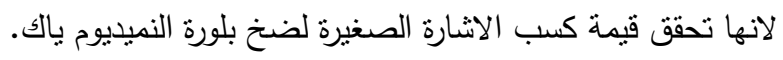




\section{Introduction}

Solar radiation as a renewable energy source can be converted directly or indirectly into other forms of energy, such as heat electricity and pumping active media of laser system [1, 2]. Solar pumped lasers convert broadband solar radiation into coherent and narrowband laser emissions [3]. Solar concentrators increase the amount of incident energy on the absorber surface as compared to that on the concentrator aperture. The increase is achieved by the use of reflecting or refracting surfaces which concentrate the incident radiation onto a suitable absorber/ receiver. Due to the apparent motion of the sun, the concentrating surface is unable to redirect the sun rays on the absorber throughout the day if both the concentrating surface and the absorber surface are stationary. Several researchers have investigated lasers powered directly from solar energy. Figure (1) shows a schematic design of a system generating the solar laser using the side pumping technique.

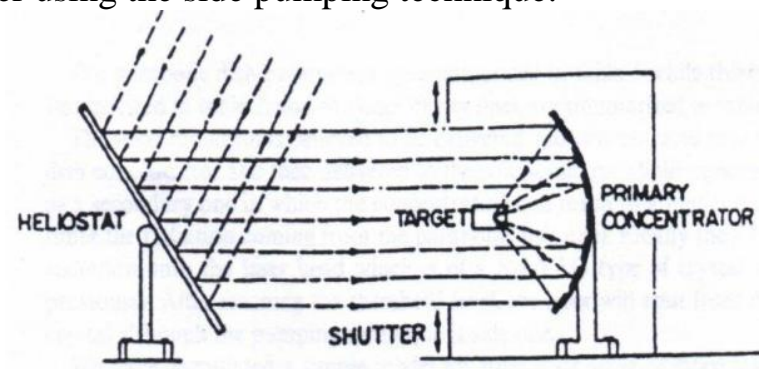

Figure (1) solar pumped laser setup [4].

\section{Concept of solar concentration}

Many concentrator types are possible for increasing the flux of the radiation on the receivers; they can be reflectors or refractors. They can be cylindrical to focus on a "line" or circular to focus on a "point". Receivers can be concave, flat or convex. These show there are two types Fresnel lens and parabola dish configurations which are shown in Figure (2).
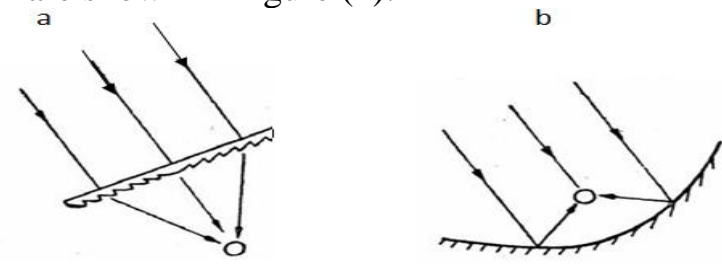

Figure (2) types of concentrator: (a) Fresnel lens (FL) (b) Parabolic collector.
There are two natural definitions of solar concentration ratio that have been in use; The first definition is strictly geometrical and named "Geometric Concentration" or "Area Concentration", while the second one is in terms of the ratio of the measured intensity and named "Intensity Concentration" or "Flux Concentration". The Area Concentration $\left(C_{\text {area }}\right)$ is the ratio of the effective area of the aperture to the surface area of the absorber [4]:

$C_{\text {area }}=\frac{A_{a}}{A_{r}}$

Where $A_{a}$ is the area of the aperture and $A_{r}$ is the area of the receiver (the absorber) of the concentrator. The flux concentration $\left(C_{f l u x}\right)$ is the ratio of the intensity at the aperture to that at the absorber [4].

$C_{f l u x}=\frac{I_{a}}{I_{r}}$

Where $I_{a}$ is the value of flux at the aperture and $I_{r}$ is the value of the flux at the receiver (the absorber) of the concentrator [5].

\section{Solar pumping \& SSG}

The relationship between solar concentration ratio $C_{\text {area }}$ and small signal gain laser, $\mathrm{g}_{\mathrm{o}}$, is obtained by [6].

$g_{o}=\sigma N W_{P} t s_{p} C_{\text {area }}$

Table (1) shows optical properties of the Nd/YAG [7]

Doping density (Nd)

Stimulated emission cross section

Lifetime of upper laser level

Pumping Rate

$\begin{array}{rll}N & {[1 / \mathrm{cm} 3]} & 1.0 \times 10^{20} \\ \sigma & {\left[\mathrm{cm}^{2}\right]} & 3.2 \times 10^{-19} \\ t_{s p} & {[\mu s]} & 240 \\ W_{p} & {[1 / \mathrm{s}]} & 1.12 \times 10^{-3}\end{array}$

For CW pumped Nd: YAG lasers the gain coefficient is typically on the order of $\mathrm{g}_{\mathrm{o}}=(0.05-0.1) \mathrm{cm}^{-1}$ and the optimum output coupling ranges $(0.80-0.98)$ [7]. The laser output power can be written as [7]:

$$
P_{\text {out }}=\left(\frac{\pi D^{2} I_{S}}{4}\right)\left(\frac{T}{(2 L-\ln (R))}\right)\left(g_{0}-(L-\ln (\sqrt{R}))\right)
$$

Where $I_{s}$ is the saturation flux, $D$ is rod diameter; $T$ and $R$ are the output mirror transmission and reflectivity, 
respectively; and $L$ is the loss per pass in the laser active media [4].

\section{$\underline{\text { Experimental Measurement }}$}

A- We measured ratio of the effective area of the Fresnel lens aperture $\left(729 \mathrm{~cm}^{2}\right)$ to the surface area of the absorber represented spot size diameter $(15,10, \ldots)$ $\mathrm{mm}$ in optical screens, as shown in Figure( 3 ).

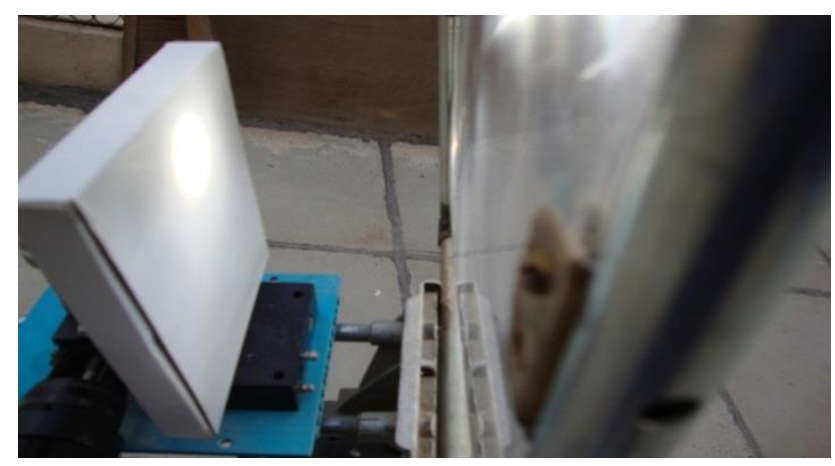

Figure (3) experiment on natural sunlight with Fresnel lens.

B-Repeat this test, we measured ratio of the effective area of the dish aperture $\left(7065 \mathrm{~cm}^{2}\right)$ to the surface area of the absorber represented spot size diameter $(60,50$, $40, \ldots) \mathrm{mm}$, as shown in Figure(4).

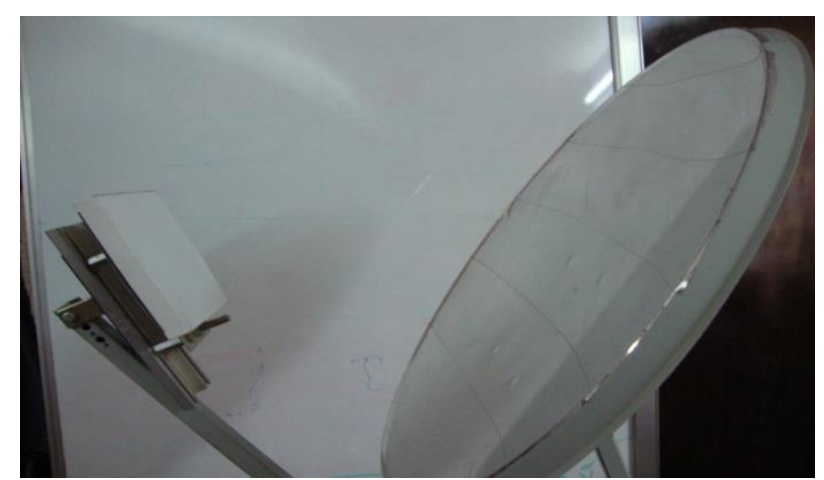

Figure (4) experiment on natural sunlight with dish.

\section{$\underline{\text { Results and discussion }}$}

The system set-up of Fresnel lens is illustrated in figure (3).The experiment was done on a clear day at which the solar flux was approximately $800 \mathrm{~W} / \mathrm{m}^{2}$. The relationship of the measured solar concentrated ratio versus spot size diameter is plotted in figure (5).

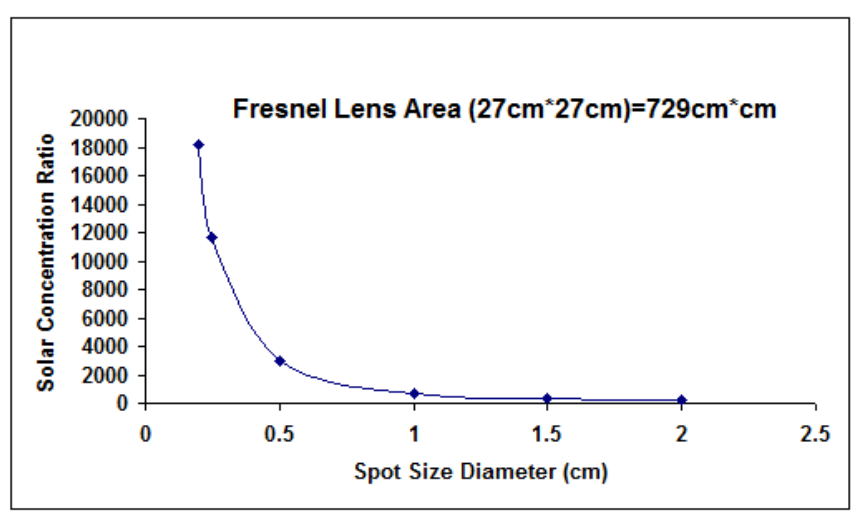

Figure (5) relationship of the measured solar concentrated ratio versus spot size diameter in Fresnel lens.

From Figure (5), the highest value of the solar concentrated ratio is achieved when the spot size diameter is smaller and less valuable when a large spot size diameter .From here we conclude that the solar concentration ratio can be increased by reducing the spot size diameter and increase the area of the Fresnel lens. When we used the dish instead of the lens the results were less important, although that the area of the dish is greater than the Fresnel lens. Figure (6) shows that the best value of the solar concentrated ratio up to (1000) and this value is not acceptable for the pumping Nd:YAG crystal.

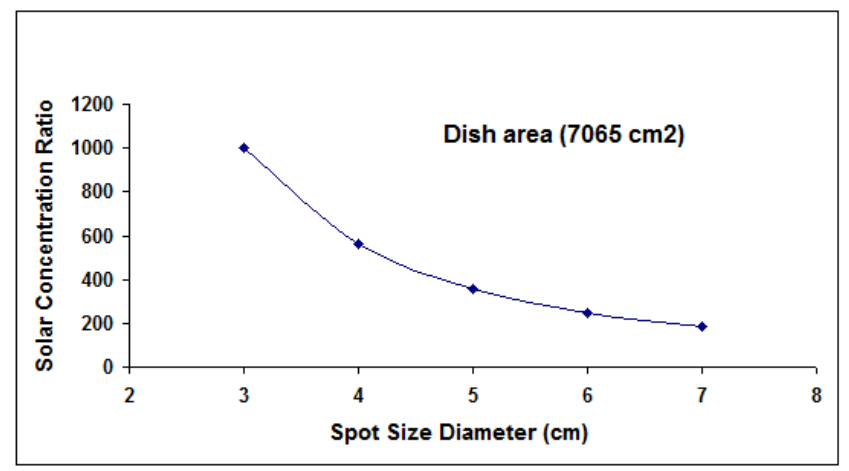

Figure (6) relationship of the measured solar concentration ratio versus spot size diameter in dish.

From the study of the relationship between the solar concentration ratios of Fresnel lens with small signal gain as represented in Figure (7). It is found that the values are acceptable for pumping laser active medium. 


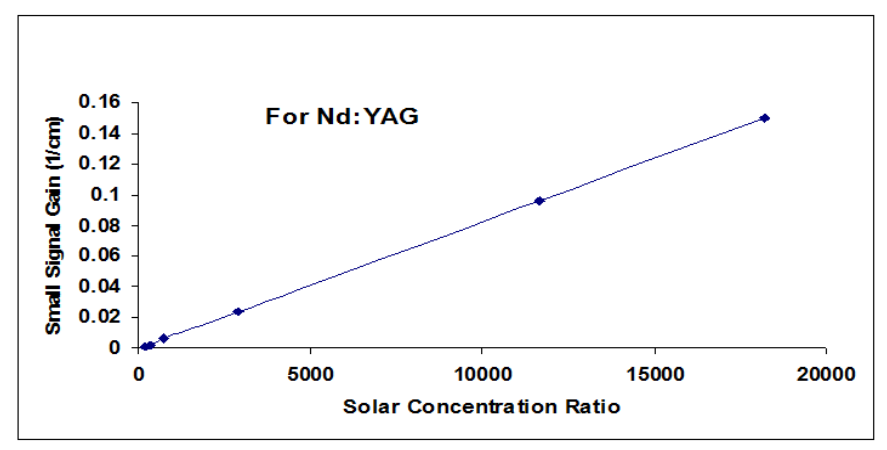

Figure (7) relationship of the measured solar concentration ratios for Fresnel lens With small signal

The relationship between the solar concentration ratios of dish with small signal gain is represented in Figure (8). This result is not acceptable for laser, because of these values are too small compared with the required values.

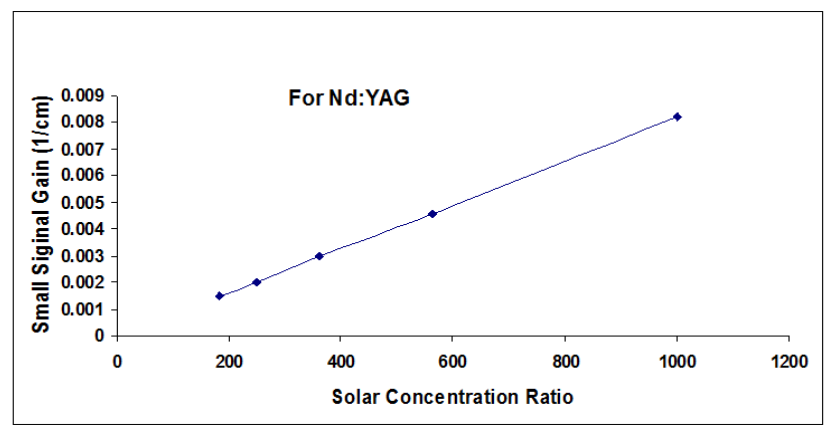

Figure (8) relationship of measured solar concentration ratios for dish versus small signal gain.

The laser output power from equation (4) under $\mathrm{L}$ is the loss per pass in the laser $0.025 \mathrm{~cm}^{-1}$ with small signal gain. Best values for the laser when solar concentration ratio is high. We conclude that the value of laser output depend on type, size of concentrator and active medium, see figure (9).

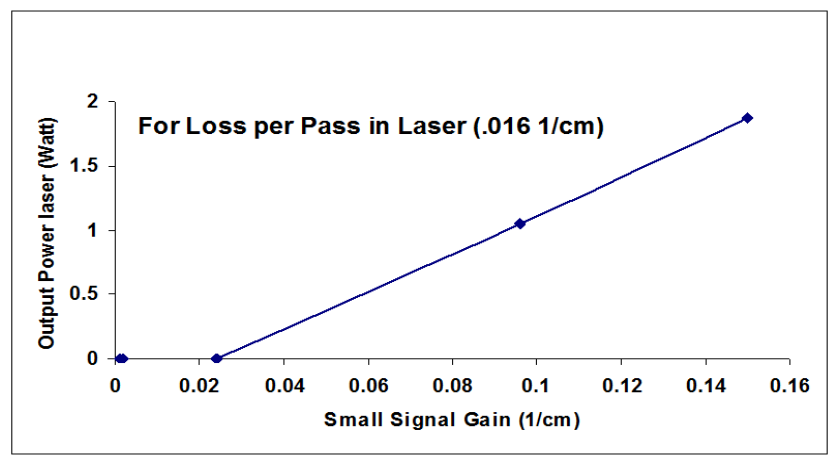

Figure (9) relationship of measured small signal gain for Frensel lens versus output power.

\section{Conclusions}

In this research, we studied more than a model for concentrating solar flux; namely solar dish and Fresnel lens. Small signal gain is calculated for Nd:YAG crystal by using the two types of solar concentrators in question. The results are good for the Fresnel lens, but not for the solar dish in spite of its bigger area which its concentration ratio is 1000 times only. First concentrator; namely the Frensel lens is necessary to increase pumped power for laser medium because it has the ability to increase solar concentration ratio to 18000 times, hence increasing small signal gain for laser medium to $0.14 \mathrm{~cm}^{-1}$ reaching the required values; namely $(0.05-0.1) \mathrm{cm}^{-1}$.

\section{References}

[1] T.Yabe, B.Bagheri, et al: Journal of Applied Physics 104,083104, 2008.

[2] T. Ohkubo, T. Yabe, et al: Optics Letters. 34, 2, January 15, 2009.

[3] C. G. Young, Appl. Opt. 5, 993, 1966.

[4] M.Weksler, J.Shwartz: IEEE J. Quantum Electronics.24, 6, 1988.

[5] H P Garg, Solar Energy Fundamentals and Applications 2000.

[6] M. Endo: Optics Express.15, 9, 2007.

[7]W. Koechner, Solid-State Laser Engineering Springer, New York, 1992. 